\title{
Beyond blueprints
}

\section{Renewed interest in the biological significance and applied outcomes of metabolism is moving the field from static biochemical charts to multidimensional networks.}

The challenges in early metabolism researchas reflected in the many Nobel prizes accorded to the field from the 1920s to the 1950s-were structural issues: identifying the relevant molecules, the enzymatic principles behind their chemical transformations and their interconnections. Although these early studies were successful in drawing the basic blueprints of basal metabolism, our understanding of the intricate wiring within these plans and their ability to change in time and space continues to evolve. Coupled with a growing awareness of its functional importance in basic and applied sciences, metabolism is reemerging as a central topic in chemistry and biology. In this focus issue, we feature a collection of commentary and review articles that outline some of the ideas, advances and goals that are laying the foundations for the next era of metabolism research.

Why has metabolism research lain dormant for so long? With initial pathway maps in place and in the absence of techniques necessary for the high-resolution study of small molecules in cells (Nature, 455, 697-700, 2008), attention turned to more tractable topics in molecular biology and genetics. Yet, as biological investigations progress toward molecular-level resolution, the answers increasingly point to metabolism as a critical component of cellular function. For example, in the immune system, metabolism is thought to serve as a determinant of macrophage phenotype (Cell Metab. 15, 432-437, 2012) and to influence the memory of T cells (Nature 460, 103-107, 2009), whereas, in development, lipid metabolism has been shown to influence Caenorhabditis elegans lifespan (Research Highlights, p. 503). In medicine, the full importance of Otto Warburg's work on the differential metabolism of cancer cells, recognized with a Nobel prize in 1931, remains under active investigation (Cancer Cell 21, 297-308, 2012). Similarly, the increasing prevalence of obesity and metabolic disease is leading scientists to ask more sophisticated questions about metabolic crosstalk within the microbiome and molecular flux across integrated, organismlevel metabolic pathways.

Amid these biological advances, chemists and biochemists have not been idle.

Explanations of long-standing questions, such as how cells switch from glucose utilization to fatty acid oxidation (J. Biol. Chem. 286,
11937-11950, 2011) or respond immediately to the loss of glucose (Article, p. 562), have highlighted new mechanisms in metabolic regulation. Increased exploration of enzyme promiscuity has demonstrated unexpected ways to make or use metabolites, whereas new post-translational modifications have revealed surprising roles for these molecules (Nat. Chem. Biol. 5, 879-881, 2009; Nat. Chem. Biol. $7,58-63,2011)$. Improvements to analytical techniques are yielding unprecedented quantities of data that can inspire new hypotheses and inform previously intractable questions (Science 335, 1099-1103, 2012).

In parallel, scientists have been inspired to rationally manipulate metabolism to achieve practical goals. These efforts in metabolic engineering to generate drugs, commodity chemicals and fuels have spurred both scientific interest and funding while simultaneously providing practical motivations for understanding not only how enzymes work but also how pathways are regulated and intertwined. For example, cells regulate intracellular metabolism via substrate channeling, enzyme assembly and compartmentalization, and cellular conglomerates can distribute tasks to increase efficiency (Review, p. 527). Further insights into the mechanisms by which enzyme localization affects activity and how complex compartments assemble can be put to use in engineering approaches (Research Highlights, p. 502, p. 503). The application of robust systems-level engineering strategies, including the integration of omics data and computational modeling, will similarly enable researchers to better understand how cellular metabolism responds to changing environmental conditions and how metabolic flux can be directed in productive ways (Review, p. 536).

In addition to co-opting metabolic pathways for applied purposes, engineers can provide important insights into the basic chemical principles and biological regulation of metabolism. For example, studies in protein design offer a stringent test of our understanding of enzyme catalysis and molecular recognition, with appropriately engineered enzymes serving to create new molecules and new pathways (Review, p. 518). Similarly, scientists can better rationalize artificial pathways and appreciate their impact on host machinery by considering how biochemical constraints and metabolic aims shape both familiar and new metabolic pathways (Review, p. 509; Nature 466, 774-778, 2010).

Despite the exciting opportunities now available, decades of disinterest have limited the number of scientists with appropriate training to take the field of metabolism forward. Educational pedagogies that focus on recurrent biochemical themes and highlight open questions in the field can help to inspire upcoming scientists to view metabolism as more than merely reactions to be memorized (Commentary, p. 497). Similarly, engineering competitions provide opportunities for students to not only engage in topics at the forefront of the field but also contribute to 'real' research, providing them with motivation for the subsequent study of relevant reactions and concepts. For example, the 2009 iGEM team TorontoMaRSDiscovery was an early pioneer in reaction compartmentalization with their 'encapsulator' (http://2009.igem. org/Team:TorontoMaRSDiscovery). The 2010 iGEM and 2011 BIOMOD team from the University of Slovenia helped to establish concepts in DNA-mediated protein assembly to speed multienzyme reactions (http://2010.igem.org/Team:Slovenia, http:// openwetware.org/wiki/Biomod/2011/ Slovenia/BioNanoWizards). And the grand prize winner of iGEM in 2011, from the University of Washington, made new inroads in biofuel production and sugar degradation (http://2011.igem.org/Team:Washington).

How can chemical biologists get involved? Each of the review articles in this focus issue outlines open questions and limitations to current technologies that can inspire future efforts. Chemical biologists can also use their interdisciplinary prowess to forge connections among colleagues, including chemical engineers, who have expertise in optimizing product yield, and plant biologists, to whom the concept of compartmentalized metabolism is already familiar. As suggested by Steven McKnight, "The more sticky problems that required attention to the dynamics of metabolism and that were pushed aside for decades now loom as interesting and important challenges" (Science 330, 1338-1339, 2010). Chemical biologists who are conversant in the chemicals of the cell are poised to answer these challenges as they draft the next set of plans. 